\title{
Angiopoietin-2 outperforms other endothelial biomarkers associated with severe acute kidney injury in patients with severe sepsis and respiratory failure
}

Wen-Kuang Yu ${ }^{1,2,3}$, J. Brennan McNeil ${ }^{3}$, Nancy E. Wickersham ${ }^{3}$, Ciara M. Shaver ${ }^{3}$, Julie A. Bastarache ${ }^{3,4,5}$ and Lorraine B. Ware ${ }^{3,5^{*}}$ (D)

\begin{abstract}
Background: Endothelial dysfunction and injury is a major pathophysiologic feature of sepsis. Sepsis is also the most frequent cause of acute kidney injury (AKI) in critically ill patients. Though most studies of AKI in sepsis have focused on tubular epithelial injury, the role of endothelial dysfunction and injury is less well studied. The goal of this study was first to investigate whether endothelial dysfunction and injury biomarkers were associated with severe AKI in sepsis patients. The second goal was to determine the best performing biomarker for severe AKI and whether this biomarker was associated with severe AKI across different etiologies of sepsis and clinical outcomes.
\end{abstract}

Methods: We studied adults with severe sepsis and acute respiratory failure (ARF) enrolled in the prospective observational Validating Acute Lung Injury markers for Diagnosis (VALID) study. Plasma endothelial dysfunction and injury biomarkers, including angiopoietin-2, soluble vascular endothelial cadherin (sVE-cadherin), endocan and syndecan-1, were measured at study enrollment. Primary analysis focused on the association between endothelial biomarker levels with severe AKI (defined as Kidney Disease: Improving Global Outcomes [KDIGO] AKI stage 2 or 3), other organ dysfunctions (defined by Brussels organ failure scores), and comparison of pulmonary versus non-pulmonary sepsis.

Results: Among 228 sepsis patients enrolled, 141 developed severe AKI. Plasma levels of angiopoietin-2, endocan, sVE-cadherin, and syndecan-1 were significantly higher in sepsis patients with severe AKI compared to those without severe AKI. Among four endothelial biomarkers, only angiopoietin-2 was independently associated with severe AKI (odds ratio 6.07 per log increase, $95 \% \mathrm{Cl}$ 2.34-15.78, $p<0.001$ ). Plasma angiopoietin-2 levels by quartile were significantly higher in sepsis patients with hepatic, coagulation, and circulatory failure. Plasma angiopoietin-2 levels were also significantly higher in patients with non-pulmonary sepsis compared to subjects with pulmonary sepsis.

Conclusion: Among four biomarkers of endothelial dysfunction and injury, angiopoietin-2 had the most robust independent association with development of severe AKI in patients with severe sepsis and ARF. Plasma angiopoietin-2 levels were also associated with other organ dysfunctions, non-pulmonary sepsis, and death. These findings highlight the importance of early endothelial dysfunction and injury in the pathogenesis of sepsis-induced AKI.

\footnotetext{
*Correspondence: lorraine.ware@vumc.org

${ }^{3}$ Division of Allergy, Pulmonary and Critical Care Medicine, Department of Medicine, Vanderbilt University Medical Center, T1218 MCN, 1161 21st, Avenue S, Nashville, TN 37232, USA

Full list of author information is available at the end of the article
}

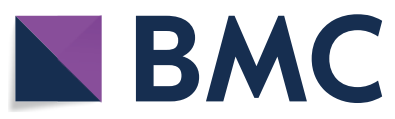

(c) The Author(s) 2021. Open Access This article is licensed under a Creative Commons Attribution 4.0 International License, which permits use, sharing, adaptation, distribution and reproduction in any medium or format, as long as you give appropriate credit to the original author(s) and the source, provide a link to the Creative Commons licence, and indicate if changes were made. The images or other third party material in this article are included in the article's Creative Commons licence, unless indicated otherwise in a credit line to the material. If material is not included in the article's Creative Commons licence and your intended use is not permitted by statutory regulation or exceeds the permitted use, you will need to obtain permission directly from the copyright holder. To view a copy of this licence, visit http://creativecommons.org/licenses/by/4.0/. The Creative Commons Public Domain Dedication waiver (http://creativeco mmons.org/publicdomain/zero/1.0/) applies to the data made available in this article, unless otherwise stated in a credit line to the data. 
Keywords: Endothelial dysfunction and injury, Sepsis, Angiopoietin-2, Acute kidney injury

\section{Introduction}

Sepsis is a syndrome of life-threatening organ dysfunction caused by a dysregulated host response to various infections [1-3]. Although numerous treatments have been studied, sepsis remains one of the most common causes of complications and death among critically ill patients admitted to the intensive care unit (ICU) $[4,5]$. The etiology is complex, including both pathogen and host factors, with sites of infection (pulmonary versus non-pulmonary) conferring additional heterogeneity [1, 4]. Endothelial dysfunction and injury plays a fundamental role in the pathogenesis of sepsis, leading to breakdown of the microvascular barrier, loss of homeostasis, and activation of the host innate immune system, culminating in intravascular fluid leakage, interstitial edema, thrombosis formation, vascular dilatation, tissue hypoperfusion, organ dysfunction, and death [6-9].

Acute kidney injury (AKI), an abrupt decrease in renal function as measured by an increase in serum creatinine and/or decrease in urine output, is commonly encountered in the ICU. Sepsis is the most frequent cause of AKI in critically ill patients [10-12], and development of AKI in septic patients results in longer ICU stays, increased costs of hospitalization, and significant long-term morbidity and mortality, especially if the AKI is severe [1315]. Early detection and aggressive treatment are of vital importance to prevent adverse outcomes. Many studies have focused on the role of acute tubular epithelial injury in sepsis-induced AKI, but the role of endothelial dysfunction and injury is less well understood $[16,17]$.

Biomarkers of endothelial dysfunction and injury have been extensively studied in the prediction of prognosis and outcomes in critically ill patients with sepsis [18-21]. However, the association between endothelial dysfunction and injury biomarkers and severe AKI in patients with sepsis has not been well studied. The first aim of this study was to explore whether commonly measured endothelial dysfunction and injury biomarkers are associated with severe AKI in patients with sepsis. The second aim was to determine the best performing biomarker for severe AKI and whether this biomarker was independently associated with severe AKI across different etiologies of sepsis and clinical outcomes.

\section{Methods}

\section{Study design and patient selection}

This was a prospective study of patients with age $\geq 18$ and severe sepsis as defined by sepsis-2 criteria [22] who were enrolled in the Validating Acute Lung Injury markers for Diagnosis (VALID) study. VALID is a singlecenter prospective observational cohort study, which has enrolled critically ill patients admitted to four ICUs (surgical, medical, trauma, and cardiovascular) at Vanderbilt University Medical Center (VUMC) since 2006 [23, 24]. Patients were enrolled in the VALID study within $24 \mathrm{~h}$ of ICU admission, if there was no plan to discharge from the ICU that day. Clinical data for enrollment day were collected within $24 \mathrm{~h}$ prior to enrollment and for the subsequent $72 \mathrm{~h}$ for a total of four study days. Patients were excluded from VALID if they had cardiac arrest prior to enrollment, uncomplicated drug overdose, or chronic lung disease requiring home oxygen therapy. After enrollment, plasma was obtained for biomarker measurement on the morning of ICU day 2, within $24 \mathrm{~h}$ of ICU admission. Additionally, clinical data and outcomes including patient demographics, Acute Physiology and Chronic Health Evaluation II (APACHE II) scores [25], medical histories, prehospital medications, admission diagnoses, etiology of sepsis (pulmonary vs. non-pulmonary), length of ICU and hospital stay, duration of mechanical ventilation, and hospital mortality were collected comprehensively. Laboratory values, hemodynamic variables, ventilator settings, in-hospital medications, fluid balance, AKI, acute respiratory distress syndrome (ARDS), and evidence of organ failures according to Brussels organ failure scores [26] were recorded for each study day. The study protocol was approved by Vanderbilt Institutional Review Board (IRB \#051065). Informed consent was obtained from the patients or surrogates whenever possible; if patients or surrogates were unable to provide consent, the institutional review board granted a waiver of consent due to the minimal risk of the study protocol.

In the current study, to enrich for likelihood of development of severe AKI, patients enrolled in VALID were included if they had severe sepsis according to the sepsis- 2 criteria, APACHE II scores equal to or greater than 25 , respiratory failure requiring mechanical ventilation, and ICU stay for at least 2 days. For analysis of AKI biomarkers, sepsis patients with known end-stage renal disease requiring regular dialysis were excluded. Patients with pneumonia or aspiration of gastric contents were grouped as pulmonary sepsis, and the remaining patients were grouped as non-pulmonary sepsis. Some patients included in the current study were also included in prior studies of syndecan-1 and soluble vascular endothelial cadherin (sVE-cadherin) levels in severe sepsis [3, 27]. 
Definitions of AKI, ARDS, and non-pulmonary organ failure AKI was determined for each study day using the Kidney Disease: Improving Global Outcomes (KDIGO) serum creatinine criteria during the study period [10]. Briefly, AKI was diagnosed as an increase in serum creatinine $\geq 0.3 \mathrm{mg} / \mathrm{dl}$ within $48 \mathrm{~h}$ or $\geq 50 \%$ times baseline. The baseline creatinine value was determined as the lowest recorded value from the medical record within one year before ICU admission. For patients with no recorded creatinine values in the previous year $(n=109)$, we used the following equation to calculate the assumption of baseline creatinine: [creatinine (in milligrams per deciliter) $=0.74-0.2$ (if patient is female) +0.08 (if patient is African ancestry) $+0.003 \times$ age (in years) $[28,29]$. The severity of AKI was determined for each study day by the degree of serum creatinine increase according to the KDIGO AKI criteria. For the current study, KDIGO AKI stage 2 or 3 was categorized as severe AKI. ARDS was assessed daily during the study period using the Berlin definition by two physician investigators who reviewed chest radiographs, blood gases, and clinical data [30]. $\mathrm{SpO}_{2} / \mathrm{FiO}_{2}$ ratio was substituted for $\mathrm{PaO}_{2} / \mathrm{FiO}_{2}$ ratio for the diagnosis of ARDS if blood gas data were not available [23, 31]. Definitions of hepatic, coagulation, and circulatory failure were based on the Brussels organ failure scores [26].

\section{Measurement of angiopoietin-2, endocan, sVE-cadherin, and syndecan-1}

Biomarkers of endothelial dysfunction and injury were measured in duplicate in plasma samples collected on the morning of ICU day 2, within $24 \mathrm{~h}$ of ICU admission, using commercially available enzyme-linked immunosorbent assay (ELISA) kits according to the manufacturers' instructions: angiopoietin-2 (Item No. DY623, R\&D Systems, Minneapolis, MN, USA); endocan (Item No. ab213776, Abcam, Cambridge, CB2 0AX, UK); sVE-cadherin (Item No. DCADV0, R\&D Systems, Minneapolis, MN, USA); syndecan-1 (Item No. ab46506, Abcam, Cambridge, CB2 OAX, UK).

\section{Statistical analysis}

The primary dependent variable was development of severe AKI within the four study days. Continuous variables were reported as median with interquartile range (IQR) and were analyzed by Mann-Whitney $U$ and Kruskal-Wallis test. Categorical variables were presented as counts and percentages and were analyzed by Chi-square or Fisher's exact test. Collinearity between these four endothelial biomarkers was analyzed by Spearman rank correlation test. Variables associated with severe AKI, including endothelial dysfunction and injury biomarkers, age, gender, chronic kidney disease (CKD), hepatic failure, coagulation failure, pulmonary sepsis, vasopressor use, and APACHE II scores were included in the univariate analysis. Following univariate analysis, those variables that were found to be associated with severe AKI $(p<0.2)$ in univariate analysis were subjected to multivariate logistic regression analysis using backward elimination to identify the independent risk factors associated with severe AKI. A variable was removed when its removal would cause a change in the exposure odds ratios (ORs) of $<10 \%$ at each stage of the backward elimination procedure. Receiver operating characteristic (ROC) curves for plasma levels of angiopoietin-2, sVE-cadherin, endocan, and syndecan- 1 to predict the development of severe AKI within the four study days were plotted, and the respective areas under the ROC curves (AUC) were calculated. The association between plasma angiopoietin-2 quartile and categorical outcomes of interest was analyzed by linear-by-linear association test. A $p$-value $<0.05$ was considered to be significant. All analyses were performed using SPSS statistics version 19.0 (IBM, Armonk, NY, USA).

\section{Results}

\section{Patient characteristics and timing and severity of AKI}

There were 228 critically ill patients with severe sepsis and respiratory failure included in this study. The demographic characteristics and clinical parameters are listed in Table 1. In total, 187 (82\%) patients had AKI within the four study days. Among patients with AKI within the four study days, $141(62 \%)$ developed severe AKI (KDIGO stage 2 or 3). Patients with severe AKI had a higher APACHE II score $(p<0.001)$, a higher percentage of coagulation failure on enrollment day $(p=0.001)$, pulmonary sepsis $(p=0.006)$, and vasopressor use $(p=0.004)$ compared to sepsis patients without severe AKI. Additionally, patients with severe AKI had significantly fewer ventilator-free days $(p<0.001)$ and a higher hospital mortality rate $(p=0.001)$ compared to severe septic patients who did not develop severe AKI. The hospital mortality rates increased with increasing stage of AKI and were highest in patients with stage 3 AKI $(p<0.001$, Fig. 1a). The serum creatinine levels within the four study days were significantly higher in sepsis patients with AKI compared to those without AKI (Fig. 1b). Among the 129 patients with AKI on enrollment day, 39 (30\%) were stage 1, 38 (29\%) were stage 2 , and $52(40 \%)$ were stage 3 . Patients with AKI stage 1 on enrollment day had a higher proportion of recovery from AKI at 48 or $72 \mathrm{~h}$ after enrollment (Fig. 1c). On the other hand, a higher percentage of sepsis patients with AKI stage 3 on enrollment day remained in severe AKI status at 48 or $72 \mathrm{~h}$ after enrollment (Fig. 1c). 
Table 1 Clinical characteristics of 228 critically ill ICU patients with severe sepsis

\begin{tabular}{|c|c|c|c|}
\hline & Severe AKI $(n=141)$ & No severe AKI $(n=87)$ & $p$ value \\
\hline Age (years) & $56[45-65]$ & $55[46-66]$ & 0.945 \\
\hline Male & $78(55)$ & $39(45)$ & 0.135 \\
\hline APACHE ॥ & 35 [30-39] & $30[26-32]$ & $<0.001$ \\
\hline Creatinine (mg/dl) & $2.03[1.29-3.74]$ & $0.93[0.75-1.21]$ & $<0.001$ \\
\hline Ever smoker & $87(62)$ & $53(61)$ & 1.000 \\
\hline Chronic kidney disease & $27(19)$ & $10(12)$ & 0.143 \\
\hline Hepatic failure & $52(37)$ & $22(25)$ & 0.081 \\
\hline Coagulation failure & $50(36)$ & $14(16)$ & 0.001 \\
\hline Vasopressor use & $90(64)$ & $38(44)$ & 0.004 \\
\hline ARDS development & $69(49)$ & $42(48)$ & 1.000 \\
\hline \multicolumn{4}{|l|}{ Primary site of infection } \\
\hline Lung & $60(43)$ & $54(62)$ & 0.006 \\
\hline Abdomen & $18(13)$ & $5(6)$ & 0.113 \\
\hline Urogenital tract & $17(12)$ & $6(7)$ & 0.261 \\
\hline Skin/soft tissue/bone & $10(7)$ & $0(0)$ & 0.015 \\
\hline Endocarditis/catheter & $9(6)$ & $10(11)$ & 0.218 \\
\hline CNS/sinus & $9(6)$ & $7(8)$ & 0.790 \\
\hline Unclear and others & $18(13)$ & $5(6)$ & 0.113 \\
\hline Ventilator-free days & 9 [0-23] & $23[11-26]$ & $<0.001$ \\
\hline ICU days & $8[5-11]$ & $7[4-11]$ & 0.438 \\
\hline Hospital days & $13[8-22]$ & $11[8-22]$ & 0.416 \\
\hline Died in hospital & $63(45)$ & $20(23)$ & 0.001 \\
\hline
\end{tabular}

ICU, intensive care unit; AKI, acute kidney injury; APACHE II, Acute Physiology and Chronic Health Evaluation II; ARDS, acute respiratory distress syndrome

Chronic kidney diseases are based on the medical records

Hepatic failure and coagulation failure are defined by Brussels organ failure scores

Continuous data are expressed as median with interquartile range [IQR], and categorical data are expressed as number of patients (\%). $p$ value is analyzed by $C h i-$ square test (gender, smoking history, chronic kidney disease, primary site of infection, hepatic failure, coagulation failure, vasopressor use, ARDS development, and mortality) or Mann-Whitney U test (age, APACHE II scores, creatinine, ventilator-free days, ICU days, and hospital days)

\section{Comparison of plasma angiopoietin-2, endocan, sVE-cadherin, and syndecan-1 levels between patients with and without severe AKI}

Four different biomarkers of endothelial dysfunction and injury were measured and evaluated for their relationship with severe AKI. Patients with severe AKI within the four study days had higher plasma levels of angiopoietin-2 (6372 pg/ml, IQR 3324-12382 pg/ml vs. $3242 \mathrm{pg} /$ $\mathrm{ml}$, IQR $1833-5355 \mathrm{pg} / \mathrm{ml}, p<0.001$, Fig. 2a), sVE-cadherin (2923 ng/ml, IQR 2265-3782 ng/ml vs. $2347 \mathrm{ng} /$ $\mathrm{ml}$, IQR 2013-3337 ng/ml, $p=0.002$, Fig. 2b), endocan (2859 pg/ml, IQR 1575-5369 pg/ml vs. 2083 pg/ml, IQR 1097-4046 pg/ml, $p=0.004$, Fig. 2c), and syndecan-1 (150.9 ng/ml, IQR 83.7-347.1 ng/ml vs. $94.7 \mathrm{ng} / \mathrm{ml}, \mathrm{IQR}$ 38.4-205.8 ng/ml, $p<0.001$, Fig. 2d) compared to sepsis patients without severe AKI. The ROC curves of the four endothelial dysfunction and injury biomarkers are shown in Additional file 1: Fig. S1. Angiopoietin-2 (AUC $=0.72$, 95\% CI 0.65-0.79, $p<0.001$ ), endocan (AUC $=0.61,95 \%$ CI $0.54-0.69, p=0.004)$, sVE-cadherin $(\mathrm{AUC}=0.62,95 \%$ CI $0.55-0.70, p=0.002)$, and syndecan-1 (AUC $=0.64$,
95\% CI $0.57-0.72, p<0.001)$ all had moderate discriminative powers for severe AKI within the four study days.

\section{Endothelial dysfunction and injury biomarkers as independent risk factors associated with severe AKI}

Collinearity between these four endothelial dysfunction and injury biomarkers is shown in Additional file 2: Fig. S2. Correlations between these endothelial biomarkers were modest. To determine whether endothelial dysfunction and injury biomarkers were independently associated with severe AKI within the four study days, the plasma levels of angiopoietin-2, endocan, sVE-cadherin, and syndecan-1 were log transformed and logistic regression analyses were performed. In univariate analysis, angiopoietin-2 $(p<0.001)$, endocan $(p=0.002)$, sVEcadherin $(p=0.005)$, and syndecan-1 $(p=0.003)$ were all significantly associated with severe AKI (Table 2). Multivariate logistic regression analysis using backward elimination, including four endothelial dysfunction and injury biomarkers, gender, CKD, hepatic failure, coagulation failure, vasopressor use, pulmonary sepsis, and APACHE 

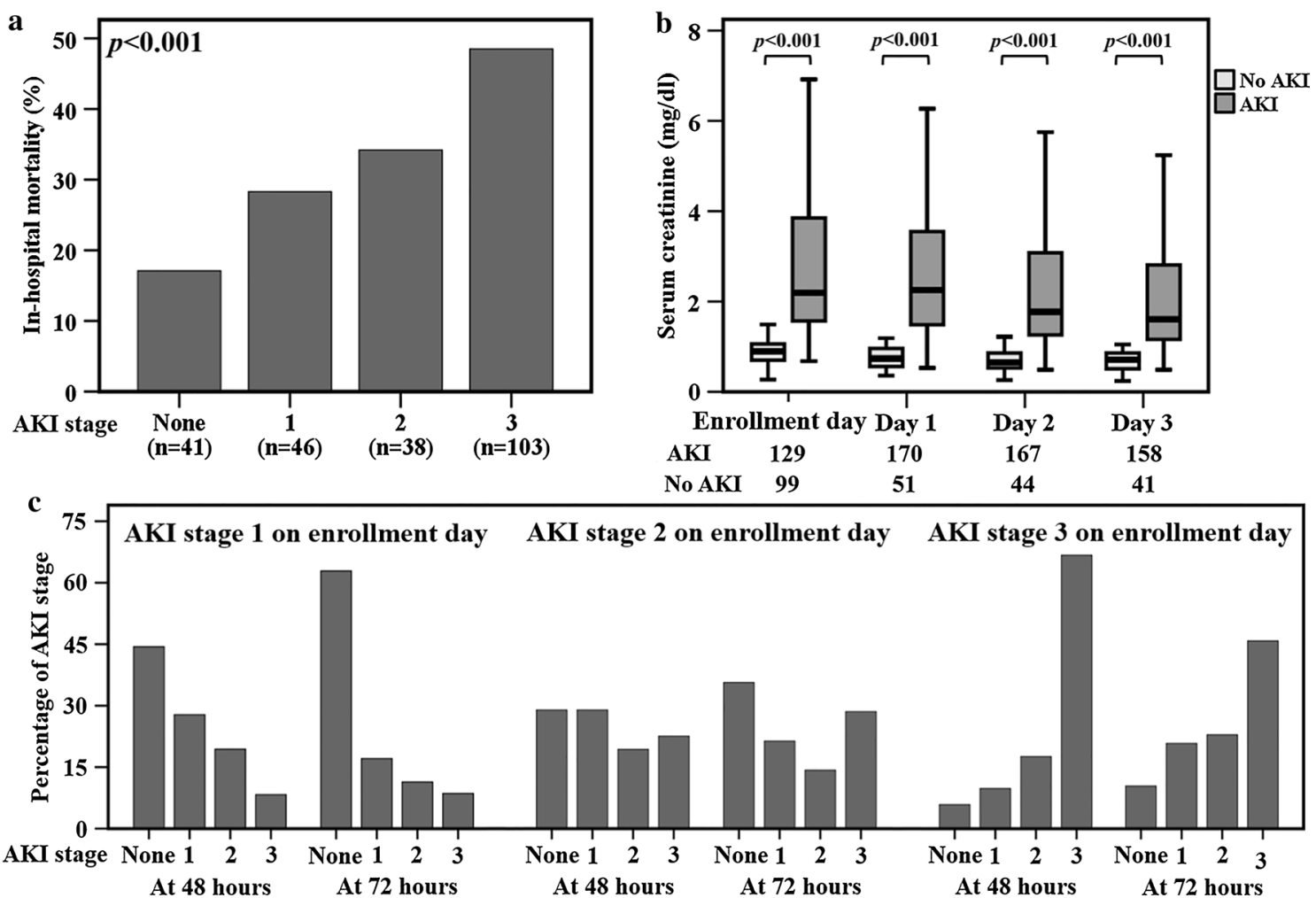

Fig. 1 a Hospital mortality rates of severe septic patients were significantly associated with severity of AKI. b Serum creatinine levels were significantly higher in sepsis with AKI compared to patients without AKI within the four study days. c Sepsis patients with AKI stage 1 on enrollment day had a higher proportion of recovery from AKI at 48 or $72 \mathrm{~h}$ after enrollment, while a higher percentage of sepsis patients with AKI stage 3 on enrollment day remained in the severe AKI status at 48 or $72 \mathrm{~h}$ after enrollment. Groups in panel a were analyzed by linear-by linear association test. Data in panel $\mathbf{b}$ were summarized as boxplots where box encompassed 25-75th percentile, error bars encompassed 10-90th percentile and horizontal line showed median. Groups in panel $\mathbf{b}$ were compared by Mann-Whitney $U$ test

II scores, showed that among the four biomarkers, only angiopoietin-2 was an independent risk factor associated with severe AKI within the four study days (odds ratio 6.07 per log increase, $95 \%$ CI 2.34-15.78, $p<0.001$, Table 2).

\section{Plasma angiopoietin-2 levels, development and resolution of AKI}

Of 228 severe sepsis patients included, 41 (18\%) had no AKI during the study period, 129 (57\%) patients had AKI on enrollment day, and 58 (25\%) patients developed AKI in the subsequent $72 \mathrm{~h}$ (Fig. 1b). The plasma levels of angiopoietin-2 were significantly lower in patients who had no AKI within the four study days $(2639 \mathrm{pg} / \mathrm{ml}$, IQR 1422-5408 pg/ml) compared to patients who developed AKI in the subsequent $72 \mathrm{~h}$ after enrollment $(4255 \mathrm{pg} /$ $\mathrm{ml}$, IQR $2656-7169 \mathrm{pg} / \mathrm{ml}$ ) or patients who had AKI on enrollment day (5844 pg/ml, IQR 2693-11,447 pg/ml, Additional file 3: Fig. S3a). Among 129 patients with AKI on enrollment day, 90 and 74 patients remained in the AKI status at 48 or $72 \mathrm{~h}$ after enrollment, respectively.
Omitting patients from the analysis whose AKI resolved by 48 or $72 \mathrm{~h}$, the plasma levels of angiopoietin-2 remained significantly lower in patients without any AKI within the four study days compared to patients with unresolved AKI at 48 (6395 pg/ml, IQR 2706-11,985 pg/ $\mathrm{ml})$ or $72(6395 \mathrm{pg} / \mathrm{ml}$, IQR $3000-11,514 \mathrm{pg} / \mathrm{ml})$ hours after enrollment (Additional file 3: Fig. S3b). Furthermore, the plasma levels of angiopoietin- 2 were significantly lower in those who had resolution of AKI in the subsequent 48 or $72 \mathrm{~h}$ compared to patients who had persistent AKI at $48 \mathrm{~h}(4221 \mathrm{pg} / \mathrm{ml}$, IQR 1854-5966 pg/ $\mathrm{ml}$ vs. $6395 \mathrm{pg} / \mathrm{ml}$, IQR $2706-11,985 \mathrm{pg} / \mathrm{ml}, p=0.027$ ) or $72 \mathrm{~h}(3805 \mathrm{pg} / \mathrm{ml}$, IQR $1744-6475 \mathrm{pg} / \mathrm{ml}$ vs. $6395 \mathrm{pg} /$ $\mathrm{ml}$, IQR 3000-11,514 pg/ml, $p=0.008$ ) after enrollment (Additional file 3: Fig. S3c).

\section{Plasma angiopoietin-2 levels, severity of AKI and cumulative fluid balance}

Because angiopoietin-2 had the strongest association with severe AKI of the biomarkers tested in the current study and was associated sepsis-induced endothelial 

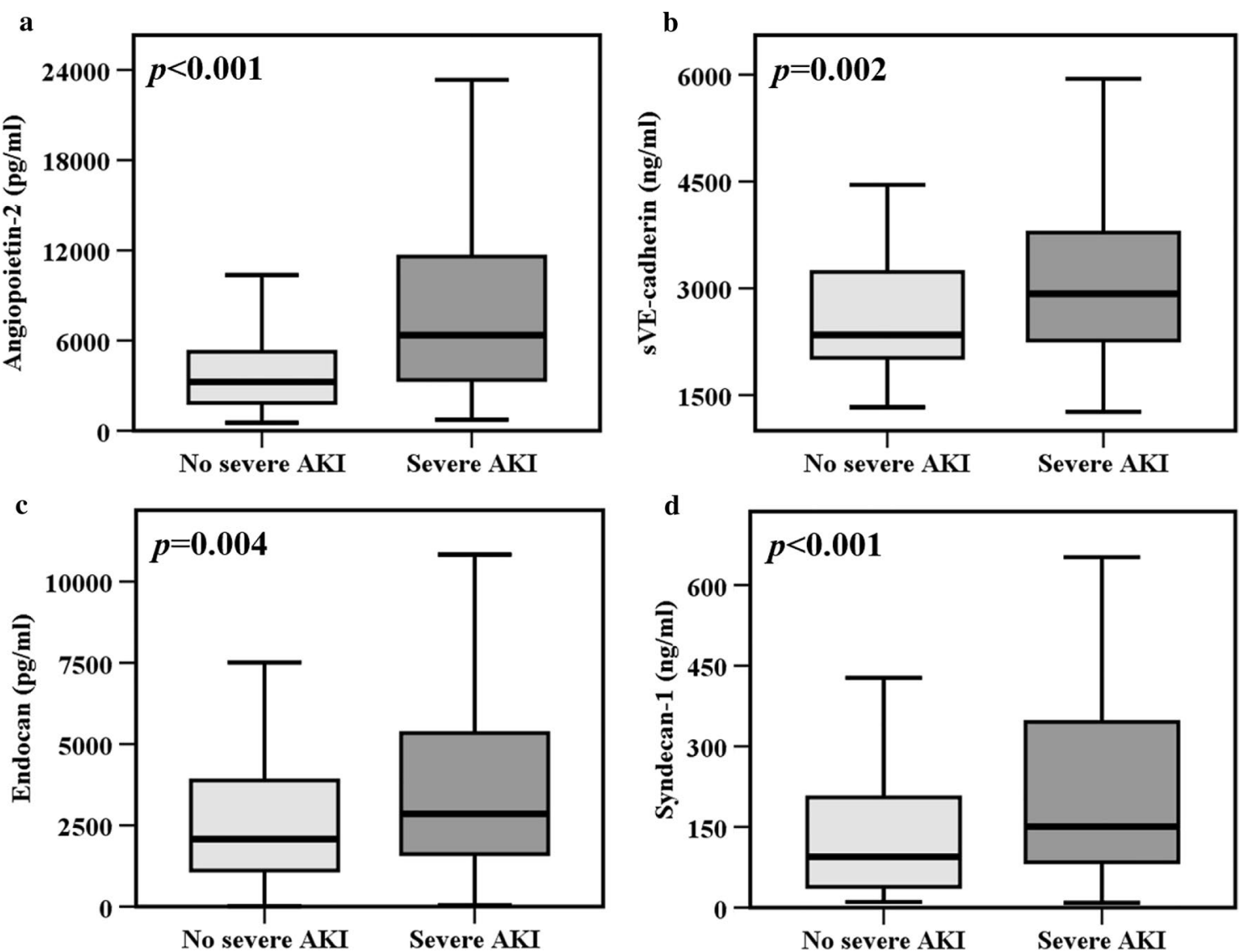

Fig. 2 Septic patients with severe AKI had significantly higher plasma levels of a angiopoietin-2, b sVE-cadherin, c endocan and $\mathbf{d}$ syndecan-1 compared to sepsis patients without severe AKI. Data in panels a-d were summarized as boxplots where box encompassed 25-75th percentile, error bars encompassed 10-90th percentile and horizontal line showed median. Groups in panels a-d were compared by Mann-Whitney $U$ test

Table 2 Univariate and multivariate logistic regression analyses of risk factors for severe AKI

\begin{tabular}{|c|c|c|c|c|}
\hline & \multicolumn{2}{|l|}{ Univariate } & \multicolumn{2}{|c|}{$\begin{array}{l}\text { Simplified model using backward LR } \\
\text { elimination method }^{\mathrm{a}}\end{array}$} \\
\hline & OR $(95 \% \mathrm{Cl})$ & $p$ value & AOR $(95 \% \mathrm{Cl})$ & $p$ value \\
\hline Male & $1.52(0.89-2.61)$ & 0.124 & $-{ }^{a}$ & \\
\hline Age & $1.00(0.98-1.02)$ & 0.993 & & \\
\hline Chronic kidney disease & $1.82(0.84-3.98)$ & 0.132 & ${ }_{-}^{a}$ & \\
\hline Vasopressor use & $2.28(1.32-3.93)$ & 0.003 & ${ }_{-}^{a}$ & \\
\hline Pulmonary sepsis & $0.45(0.26-0.78)$ & 0.004 & ${ }_{-}^{a}$ & \\
\hline Hepatic failure & $1.73(0.96-3.12)$ & 0.071 & $-{ }^{a}$ & \\
\hline Coagulation failure & $2.87(1.47-5.59)$ & 0.002 & $1.99(0.90-4.42)$ & 0.089 \\
\hline APACHE ॥ & $1.26(1.17-1.35)$ & $<0.001$ & $1.24(1.15-1.34)$ & $<0.001$ \\
\hline Angiopoietin-2 (per log increase) & $8.81(3.88-20.01)$ & $<0.001$ & $6.07(2.34-15.78)$ & $<0.001$ \\
\hline Endocan (per log increase) & $2.29(1.34-3.91)$ & 0.002 & $-{ }^{a}$ & \\
\hline sVE-cadherin (per log increase) & $11.87(2.09-67.43)$ & 0.005 & ${ }_{-}^{a}$ & \\
\hline Syndecan-1 (per log increase) & $2.73(1.32-3.93)$ & 0.003 & ${ }_{-}^{a}$ & \\
\hline
\end{tabular}

$\mathrm{OR}$, odds ratio; $\mathrm{Cl}$, confidence interval; $\mathrm{AOR}$, adjusted odds ratio

a Variables that were entered into multivariate logistic regression analysis with backward LR elimination method did not retain the final model 
permeability in previous studies $[18,35,36]$, we further analyzed the relationship between angiopoietin-2, severity of AKI and cumulative fluid balance in greater depth. Plasma angiopoietin-2 levels increased with stage of AKI and were highest in patients with stage 3 AKI $(p<0.001$, Fig. 3a). Patients who required renal replacement therapy during hospitalization had significantly higher levels of angiopoietin- 2 compared to those who did not $(p<0.001$, Fig. 3b). Higher plasma angiopoietin-2 levels by quartile at enrollment were significantly associated with a higher positive fluid balance within the four study days (Fig. 3c-f). Moreover, patients with more severe AKI had a higher cumulative fluid balance within the four study days (Additional file 4: Fig. S4).

\section{Plasma angiopoietin-2 levels, etiology of sepsis and severe AKI}

Previous studies reported that endothelial dysfunction and injury were more profound in non-pulmonary sepsis $[2,3]$. In our study, plasma angiopoietin-2 levels were also significantly higher in non-pulmonary sepsis patients compared to those with pulmonary sepsis $(p<0.001$, Fig. 4a). Next, we sought to determine whether plasma angiopoietin-2 levels were associated with severe AKI in both pulmonary and non-pulmonary sepsis. In patients with pulmonary sepsis, plasma angiopoietin-2 levels were higher in patients with severe AKI compared to those without severe AKI $(p<0.001$, Fig. $4 \mathrm{~b})$. In patients with non-pulmonary sepsis, plasma angiopoietin-2 levels were also higher in patients with severe AKI compared to those without severe AKI $(p<0.001$, Fig. $4 \mathrm{~b})$. Furthermore, plasma angiopoietin-2 levels were higher in severe AKI patients with non-pulmonary sepsis compared to those with pulmonary sepsis ( $p=0.008$, Fig. $4 \mathrm{~b})$.

\section{Plasma angiopoietin-2 levels, other organ failures and mortality}

To determine whether plasma angiopoietin-2 levels were also associated with non-kidney organ dysfunction, we compared quartiles of angiopoietin-2 levels to indices of non-kidney organ dysfunction. Higher plasma angiopoietin-2 levels by quartile were significantly associated with hepatic failure $(p<0.001$, Fig. $5 \mathrm{a})$, coagulation failure $(p<0.001$, Fig. 5 b) , and circulatory failure $(p=0.003$, Fig. 5c) as defined by Brussels organ failure scores. Plasma angiopoietin-2 levels were not associated with the development of ARDS ( $p=0.953$, Fig. 5d). Plasma angiopoietin-2 levels were significantly higher in patients with
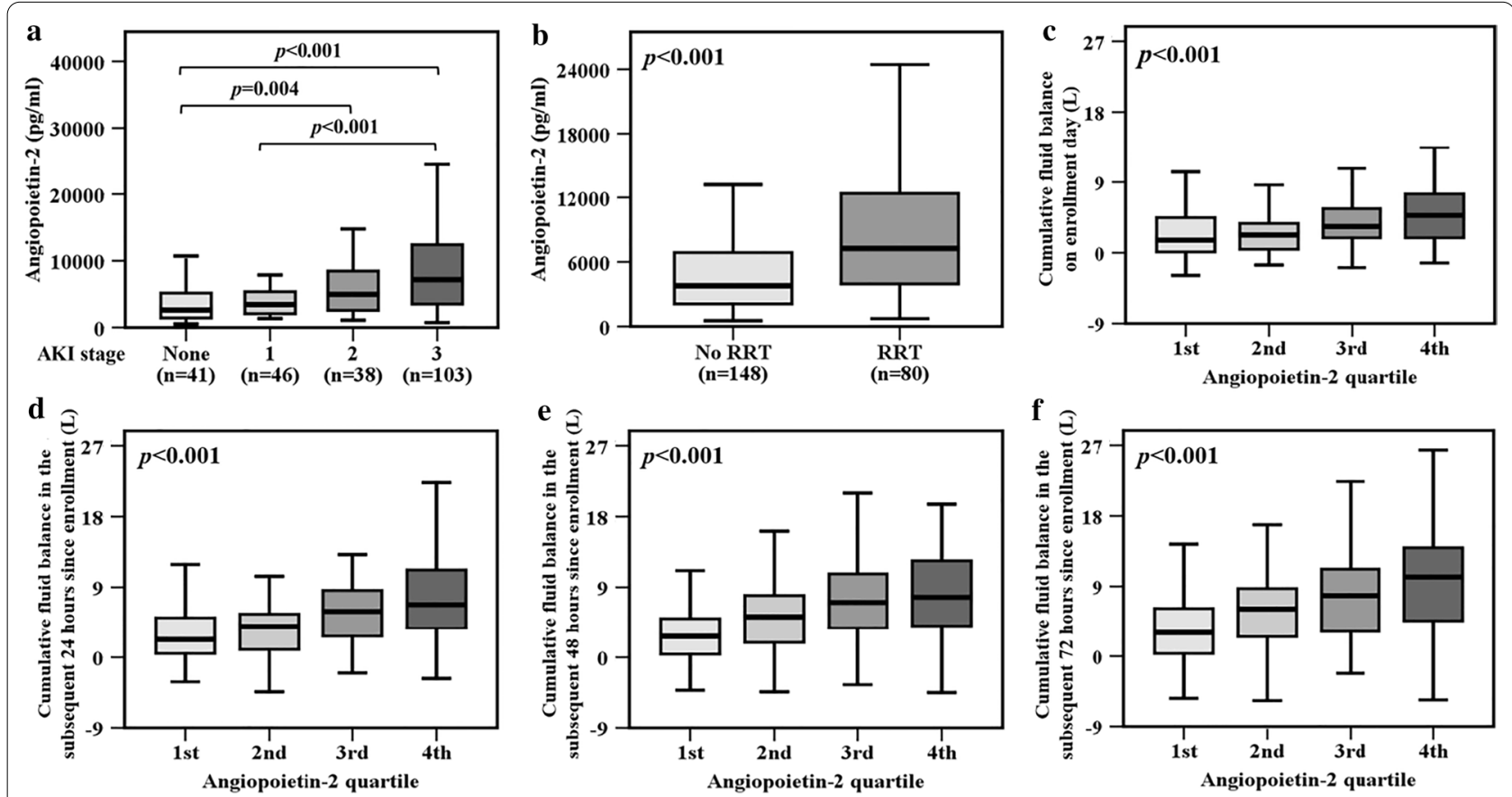

Fig. 3 a Plasma angiopoietin-2 levels were significantly associated with severity of AKI. b Patients requiring renal replacement therapy (RRT) had significantly higher plasma angiopoietin-2 levels than those not requiring RRT during hospitalization. Higher plasma angiopoietin-2 levels by quartile were significantly associated with positive fluid balance c on enrollment day, $\mathbf{d}$ in the subsequent $24 \mathrm{~h}, \mathbf{e} 48 \mathrm{~h}$ and $\mathbf{f} 72 \mathrm{~h}$ since enrollment. Data in panels a-f were summarized as boxplots where box encompassed 25-75th percentile, error bars encompassed 10-90th percentile and horizontal line showed median. Groups were compared by Kruskal-Wallis test (panels a and c-f) or Mann-Whitney $U$ test (panel b). Post hoc analysis of groups comparison was performed using Mann-Whitney $U$ test and Bonferroni correction (panel a) 

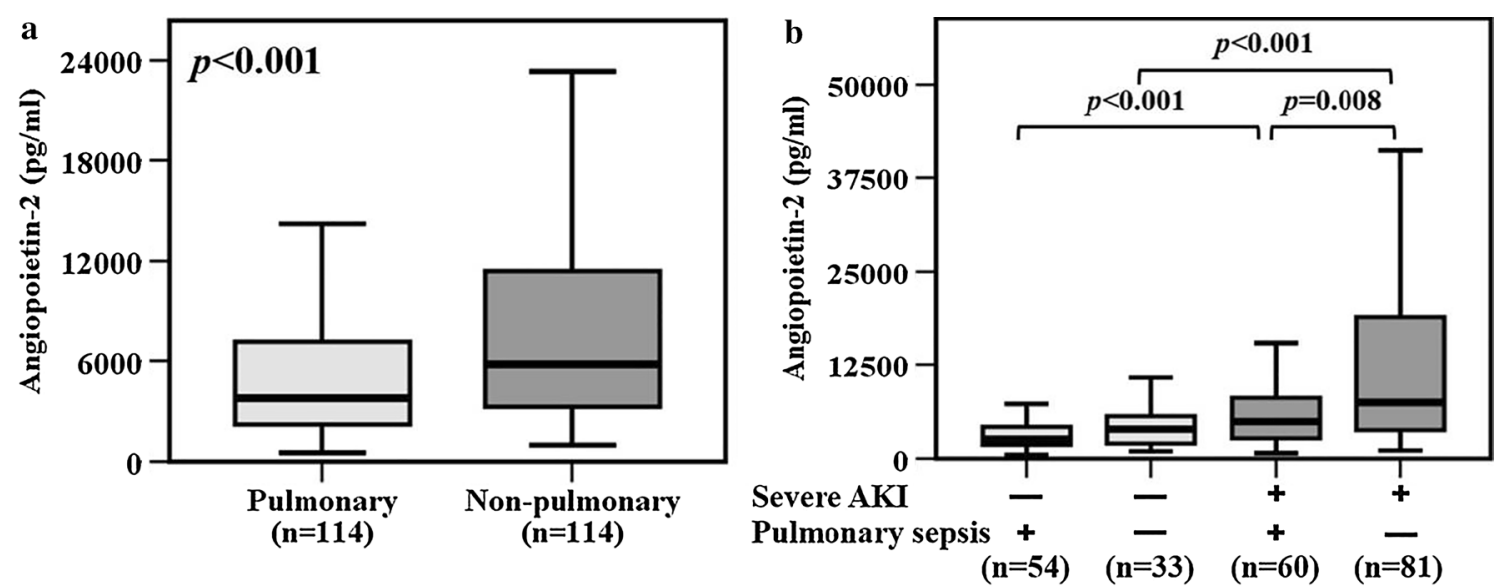

Fig. 4 a Plasma angiopoietin-2 levels were significantly higher in patients with non-pulmonary sepsis compared to those with pulmonary sepsis. b Plasma angiopoietin-2 levels were significantly higher in both pulmonary and non-pulmonary sepsis patients with any severe AKI during the study period compared to those without severe AKI during the study period. Data in panels $\mathbf{a}$ and $\mathbf{b}$ were summarized as boxplots where box encompassed 25-75th percentile, error bars encompassed 10-90th percentile and horizontal line showed median. Groups were compared by Mann-Whitney $U$ test (panel a) or Kruskal-Wallis test (panel b). Post hoc analysis of groups comparison was performed using Mann-Whitney $U$ test and Bonferroni correction (panel b)
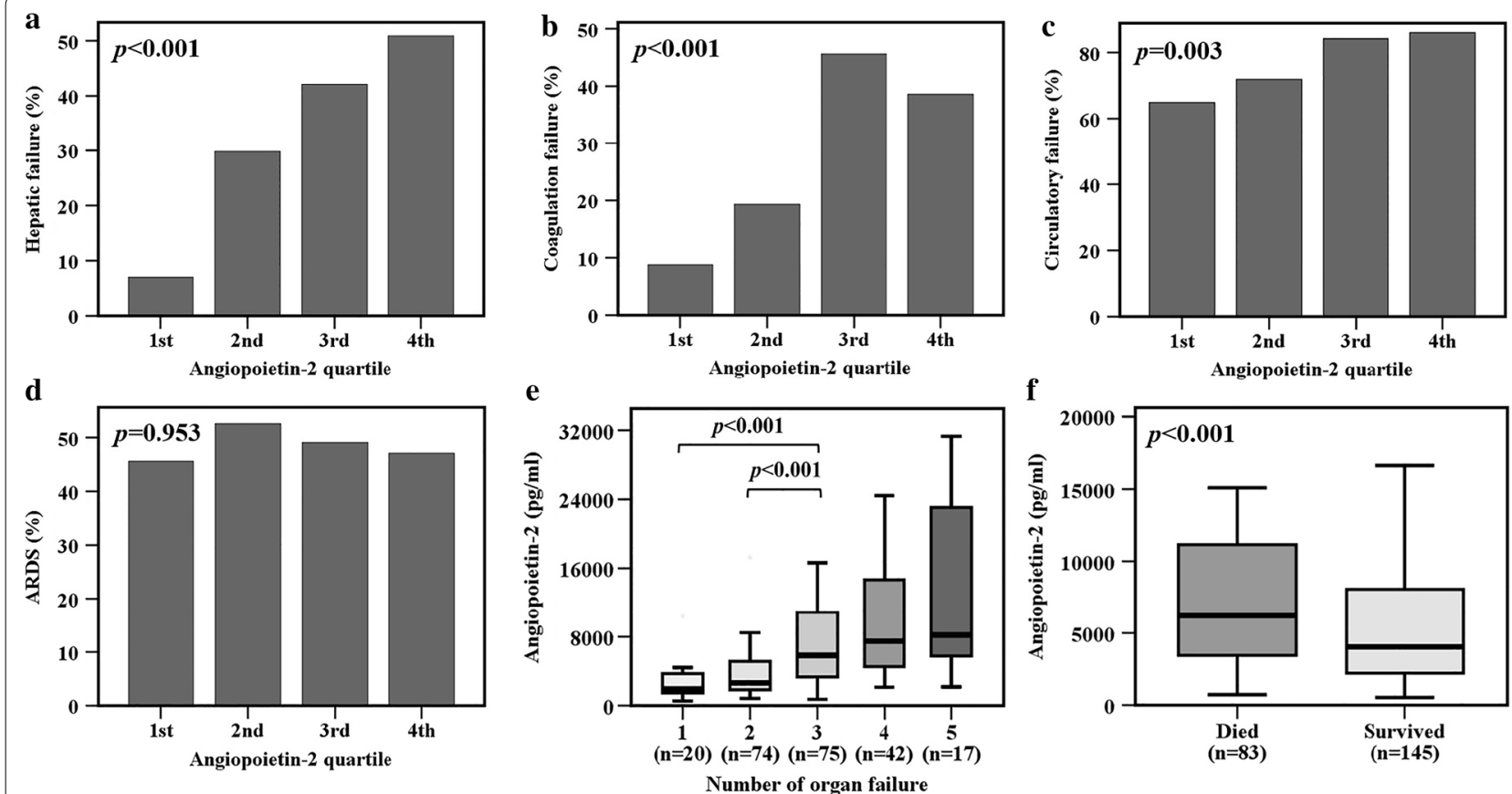

Fig. 5 Higher plasma angiopoietin-2 levels by quartile were significantly associated with $\mathbf{a}$ hepatic failure, $\mathbf{b}$ coagulation failure and $\mathbf{c}$ circulatory failure on enrollment day. Organ failures were defined by Brussels organ failure scores. $\mathbf{d}$ Plasma angiopoietin-2 levels were not associated with the development of ARDS defined by Berlin criteria during the study period. e Plasma angiopoietin-2 levels were associated with the number of organ failures on enrollment day. $\mathbf{f}$ Severe sepsis patients who died during hospitalization had significantly higher plasma angiopoietin-2 levels compared to those who survived. Groups in panels $\mathbf{a}-\mathbf{d}$ were analyzed by linear-by linear association test. Data in panels $\mathbf{e}$ and $\mathbf{f}$ were summarized as boxplots where box encompassed 25-75th percentile, error bars encompassed 10-90th percentile and horizontal line showed median. Groups were compared by Kruskal-Wallis test (panel e) or Mann-Whitney $U$ test (panel f). Post hoc analysis of groups comparison was performed using Mann-Whitney $U$ test and Bonferroni correction (panel e) 
a greater number of organ failures $(p<0.001$, Fig. 5e). Moreover, higher plasma angiopoietin-2 levels by quartile were significantly associated with higher APACHE II scores ( $p=0.008$, Additional file 5: Fig. S5). Patients who died in hospital had higher plasma angiopoietin-2 levels compared to sepsis patients who survived $(p<0.001$, Fig. 5f).

\section{Discussion}

Endothelial dysfunction and injury is a major pathophysiologic hallmark of sepsis, leading to production and release of a variety of biomarkers from the endothelium into the circulation $[8,19]$. In the present study, we found that several endothelial dysfunction and injury biomarkers, including angiopoietin-2, endocan, sVE-cadherin, and syndecan-1, were higher in sepsis patients who developed severe AKI. Of these four biomarkers, angiopoietin-2 had the most robust association with severe AKI. Furthermore, plasma angiopoietin-2 levels were significantly higher in both sepsis patients with more organ failures and those who died in the hospital. These findings indicate that sepsis-induced endothelial dysfunction and injury at ICU admission as measured by angiopoietin-2 is associated with an increased risk of adverse outcomes, including AKI, multiple organ failures, and death.

Angiopoietin-2 is produced in endothelial cells and pre-stored in the Weibel-Palade bodies together with von Willebrand factor (vWF). Kümpers et al. reported that protein expression of angiopoietin-2 was prominent in the glomerular endothelium in patients with lupus nephritis compared with healthy subjects and that the serum levels of angiopoietin- 2 were closely correlated with the severity of systemic lupus erythematosus [32]. In addition, serum levels of angiopoietin-2 were significantly elevated in ANCA-associated vasculitis with renal involvement compared to patients with active limited granulomatous disease restricted to the respiratory tract or healthy subjects [33]. Taken together, these findings suggest that angiopoietin-2 may be released from the renal endothelium when the kidneys suffer an inflammatory injury. In the setting of sepsis, angiopoietin-2 is released into the circulation where it may have autocrine effects that include disruption of the endothelial barrier and increased microvascular permeability $[8,19,34]$. AKI is one of the most common sepsis-related organ dysfunctions [10-12]. Robinson-Cohen et al. reported plasma levels of endothelial biomarkers, especially angiopoietin-2, were significantly increased in critically ill patients with AKI, independent of inflammation, and angiopoietin-2 remained associated with the development of later onset (24 h later) AKI [35]. Our present study, in concordance with previous reports, demonstrated plasma angiopoietin-2 levels at enrollment were not only significantly higher in severe sepsis patients with AKI, but also were associated with AKI severity including both KDIGO AKI stage and need for renal replacement therapy. Vascular endothelial cadherin (VE-cadherin) is the major proteinaceous component of adherens junctions between endothelial cells [3]. Endocan is a proteoglycan secreted by activated endothelium, and syndecan-1 is a proteoglycan component of the endothelial glycocalyx $[27,36]$. The results of the present study also demonstrated that increased shedding of VE-cadherin, endocan and syndecan-1 from the endothelium into the circulation is associated with severe AKI. However, in multivariable analysis, only angiopoietin-2 was independently associated with severe AKI within the four study days, even when controlling for severity of illness and other organ dysfunctions. Release of angiopoietin-2 from dysfunctional endothelial cells into the circulation may precede profound endothelial injury, including disruption of adherens junctions and shedding of endothelial proteoglycan into the circulation. A rise in plasma angiopoietin-2 levels could be a biomarker for early detection of severe AKI, but larger studies are needed.

Ziegler et al. reported that angiopoietin- 2 modulated endothelial dysfunction and injury, including microvascular permeability, in a lipopolysaccharide-induced murine sepsis model [37]. Fisher and colleagues found that higher plasma baseline levels of angiopoietin-2 were significantly associated with fluid overload, an index of microvascular permeability and leakage, within the first six hours in patients with development of septic shock [38]. Our current study further showed that plasma levels of angiopoietin-2 were not only strongly correlated with positive fluid balance within the four study days in patients with severe sepsis and respiratory failure, but also inversely associated with the ventilator-free days $(p<0.001$, Spearman's rho $=-2.33)$. These findings support the concept that angiopoietin-2 levels may be an indicator of more profound sepsis-induced microvascular permeability that results in more fluid leakage from the circulation into the interstitium, more severe pulmonary edema and requirement for longer mechanical ventilation. Kümpers et al. [39] and David et al. [40] reported circulating angiopoietin-2 levels were associated with multiple organ dysfunction, disease severity and death in sepsis patients. In the current study, we extended these findings to sepsis patients requiring mechanical ventilator support for respiratory failure and found that plasma angiopoietin-2 levels were not only correlated with the disease severity, including the number of organ failures and APACHE II scores, but also were significantly higher in sepsis patients who died in the hospital. 
Taken together, these findings reveal that the severity of endothelial dysfunction and injury is closely associated with the severity of sepsis and its outcomes.

Sepsis is a heterogeneous clinical syndrome with wide variability in both the type of underlying infection and the primary site of infection. We previously reported that VE-cadherin shedding was more severe in patients with non-pulmonary sepsis compared to those with pulmonary sepsis [3]. Murphy et al. reported that ARDS patients with non-pulmonary sepsis had higher levels of plasma syndecan-1 compared to those with pulmonary sepsis [27]. The present study adds to these prior studies, demonstrating that plasma angiopoietin-2 levels were also higher in non-pulmonary sepsis compared to pulmonary sepsis. These findings suggest that biomarkers of endothelial dysfunction and injury such as angiopoietin-2 could have a role in prognostic and predictive enrichment for future studies of sepsis therapies that focus on ameliorating endothelial dysfunction and injury in patients with non-pulmonary sepsis. The need for new approaches to reduce heterogeneity in clinical trials in sepsis and other critical illness syndrome has been identified as a research priority by the National Heart Lung and Blood Institute [41].

The pathophysiology of sepsis-induced AKI is complex and multifactorial, including inflammation, renal tubular epithelial and glomerular endothelial cellular responses to the inflammatory insult, and microvascular dysfunction $[16,17,42]$. Cross-talk with other organs, especially the lung, has also been reported to predispose to AKI in human and animal studies [43-45]. In the current study, we included only more severely ill patients with respiratory failure requiring mechanical ventilation and high severity of illness as measured by APACHE II score equal to or greater than 25 . Given the uniform presence of respiratory failure in all patients included in the current study, the role of lung-kidney cross-talk in development of severe AKI in this study cannot be determined.

Our study has several limitations. First, the VALID study is a single-center, prospective, and observational cohort study, which has enrolled critically ill patients admitted to Vanderbilt Medical, Surgical, Trauma and Cardiovascular ICUs since 2006. Treatment strategies for patients with sepsis and respiratory failure might not be consistent over a 15-year period, including mechanical ventilator settings, antibiotic use, intravenous fluid resuscitation and timing of renal replacement therapy for AKI. A larger multi-center study to measure plasma angiopoietin-2 levels should be performed to validate these results. Second, the VALID study was not designed for AKI as a primary endpoint. Hourly urine output data were not collected, and thus, AKI was only determined by changes in serum creatinine. Furthermore, 109 (48\%) patients in the current study required estimation of baseline creatinine because they had no baseline creatinine recorded in the previous year. Therefore, the incidence and severity of AKI might not be fully and exactly captured. Of note, there were no significant differences in the occurrence of severe AKI between patients with known vs estimated baseline creatinine ( $68 \%$ vs. $58 \%$, respectively, $p=0.275$ ). In addition, a large majority of patients enrolled in the current study developed AKI within $24 \mathrm{~h}$. Whether these endothelial biomarkers predict later onset of AKI is not clear and will require further study. Third, this study only enrolled severe sepsis patients with APACHE II scores equal to or greater than 25 and respiratory failure requiring mechanical ventilation. For this reason, further studies should be carried out to unravel these controversies in less severely ill septic patients, septic patients without respiratory failure, or critically ill patients without sepsis. Fourth, the mechanisms of plasma angiopoietin-2 clearance remain unclear and have not been well studied. Elevation of plasma angiopoietin-2 levels or other endothelial biomarkers might also be due to impairment of clearance in the setting of organ dysfunction. Fifth, endothelial biomarkers were only measured on the morning of ICU day 2. Whether kinetic changes in these endothelial biomarkers over time are associated with disease improvement or progression is not known. Plasma levels of angiopoietin- 2 and other endothelial biomarkers should be measured serially in future studies to clarify these issues. Finally, as this was an observational study, no conclusions about a causal role for angiopoietin-2 in the pathogenesis of sepsis-induced AKI can be made.

\section{Conclusion}

In conclusion, the present study demonstrates that among four plasma biomarkers of endothelial dysfunction and injury, the best performing biomarker independently associated with severe AKI is angiopoietin-2. Plasma angiopoietin-2 levels were also associated with hepatic failure, coagulation failure, circulatory failure and the overall number of organ dysfunctions. Patients who died and patients with non-pulmonary sepsis had higher plasma angiopoietin-2 levels compared to those who survived or who had pulmonary sepsis. Furthermore, sepsis patients with severe AKI had higher plasma angiopoietin-2 levels regardless of whether sepsis was pulmonary or non-pulmonary. Taken together, these findings suggest that endothelial dysfunction and injury as measured by circulating angiopoietin-2 levels is more extensive in sepsis patients with severe AKI. 


\section{Supplementary Information}

The online version contains supplementary material available at https://doi. org/10.1186/s13054-021-03474-z.

Additional file 1. Receiver operator curves of plasma levels of angiopoietin-2, endocan, sVE-cadherin and syndecan-1 predicted the development of severe AKI within the four study days. AUC, area under the curve.

Additional file 2. Plasma levels of angiopoietin-2 were modestly associated with (a) plasma levels of endocan, (b) plasma levels of syndecan-1 and (c) plasma levels of sVE-cadherin. Plasma levels of endocan were modestly associated with (d) plasma levels of syndecan-1, but not associated with (e) plasma levels of sVE-cadherin. Plasma levels of sVE-cadherin were modestly associated with (f) plasma levels of syndecan-1. Collinearity of these four endothelial biomarkers was analyzed by Spearman rank correlation test.

Additional file 3. (a) Plasma levels of angiopoietin-2 were significantly lower in sepsis patients who did not develop AKI within the four study days compared to patients who had AKI on enrollment day or patients who developed AKI in the subsequent 72 hours after enrollment. (b) Plasma levels of angiopoietin-2 were significantly lower in sepsis patients without any AKI within the four study days compared to sepsis patients with persistent AKl at 48 or 72 hours since enrollment. (c) Among patients with AKI on enrolment day, plasma levels of angiopoietin-2 were significantly lower in patients with the resolution of AKI compared to patients without the resolution of AKI at 48 hours or 72 hours after enrollment. Data in panels a-c were summarized as boxplots where box encompassed 25-75th percentile, error bars encompassed 10-90th percentile and horizontal line showed median. Groups were compared by KruskalWallis test (panels $\mathbf{a}$ and $\mathbf{b}$ ) or Mann-Whitney $\mathrm{U}$ test (panel c). Post hoc analysis of groups comparison was performed using Mann-Whitney $U$ test and Bonferroni correction (panels $\mathbf{a}$ and $\mathbf{b}$ ).

Additional file 4. (a) The severity of AKI within the four study days was not associated with positive cumulative balance on enrollment day. The severity of AKI within the four study days was significantly associated with positive fluid balance in the subsequent (b) 24 hours, (c) 48 hours and (d) 72 hours since enrollment. Data in panels a-d were summarized as boxplots where box encompassed 25-75th percentile, error bars encompassed 10-90th percentile and horizontal line showed median. Groups were compared by Kruskal-Wallis test (panels a-d).

Additional file 5. Higher plasma angiopoietin-2 levels by quartile were associated with higher APACHE II scores. Data were summarized as boxplots where box encompassed 25-75th percentile, error bars encompassed 10-90th percentile and horizontal line showed median. Groups were compared by Kruskal-Wallis test. Post hoc analysis of groups comparison was performed using Mann-Whitney $U$ test and Bonferroni correction.

\section{Abbreviations}

AKI: Acute kidney injury; VALID: Validating Acute Lung Injury markers for Diagnosis; ARDS: Acute respiratory distress syndrome; ICU: Intensive care unit; CKD: Chronic kidney disease; APACHE II: Acute Physiology and Chronic Health Evaluation II; KDIGO: Kidney Disease: Improving Global Outcomes; IQR: Interquartile range; ELISA: Enzyme-linked immunosorbent assay; VWF: Von Willebrand factor.

\section{Acknowledgements}

Not applicable.

\section{Authors' contributions}

W-K.Y., L.B.W., and J.A.B. conceived and designed the study; W-K.Y., J.B.M., and N.E.W. collected, processed, and analyzed samples; W-K.Y., C.M.S., J.B.M., J.A.B., and L.B.W. analyzed data and wrote the manuscript. All authors read and approved the final manuscript.

\section{Funding}

This research was funded by National Institutes of Health grants HL103836 (L.B.W.), HL135849 (J.A.B. and L.B.W.), and Taipei Veterans General
Hospital-National Yang-Ming University Excellent Scientist Cultivation Program No.107-V-A-001 (W-K.Y.)

\section{Availability of data and materials}

The data used and analyzed during the current study are available from the corresponding author on reasonable request.

\section{Ethics approval and consent to participate}

The prospective study was approved by Vanderbilt Institutional Review Board (IRB \#051065). Informed consent was obtained for sample collection from the patients or surrogates whenever possible; if patients or surrogates were unable to provide consent, the institutional review board granted a waiver of consent due to the minimal risk nature of the study.

\section{Consent for publication}

Not applicable.

\section{Competing interests}

The authors declare they have no competing interests.

\section{Author details}

${ }^{1}$ Division of Respiratory Therapy, Department of Chest Medicine, Taipei Veterans General Hospital, Number 201, Section 2, Shipai Road, Beitou District, Taipei City 11217, Taiwan, ROC. ${ }^{2}$ Institute of Physiology, National Yang-Ming University, Taipei, Taiwan. ${ }^{3}$ Division of Allergy, Pulmonary and Critical Care Medicine, Department of Medicine, Vanderbilt University Medical Center, T1218 MCN, 1161 21st, Avenue S, Nashville, TN 37232, USA. ${ }^{4}$ Department of Cell and Developmental Biology, Vanderbilt University School of Medicine, Nashville, TN, USA. ${ }^{5}$ Department of Medicine and Department of Pathology, Microbiology and Immunology, Vanderbilt University School of Medicine, T1218 MCN, 1161 21st, Avenue S, Nashville, TN 37232, USA.

Received: 29 September 2020 Accepted: 20 January 2021

Published online: 04 February 2021

\section{References}

1. Singer M, Deutschman CS, Seymour CW, Shankar-Hari M, Annane D, Bauer M, Bellomo R, Bernard GR, Chiche JD, Coopersmith CM, Hotchkiss RS, Levy MM, Marshall JC, Martin GS, Opal SM, Rubenfeld GD, van der Poll T, Vincent JL, Angus DC. The third international consensus definitions for sepsis and septic shock (sepsis-3). JAMA. 2016;315:801-10.

2. Calfee CS, Janz DR, Bernard GR, May AK, Kangelaris KN, Matthay MA, Ware LB. Distinct molecular phenotypes of direct vs indirect ARDS in singlecenter and multicenter studies. Chest. 2015:147:1539-48.

3. Yu WK, McNeil JB, Wickersham NE, Shaver CM, Bastarache JA, Ware LB. Vascular endothelial cadherin shedding is more severe in sepsis patients with severe acute kidney injury. Crit Care. 2019;23:18.

4. Angus DC, van der Poll T. Severe sepsis and septic shock. N Engl J Med. 2013:369:840-51.

5. Meyer N, Harhay MO, Small DS, Prescott HC, Bowles KH, Gaieski DF, Mikkelsen ME. Temporal trends in incidence, sepsis-related mortality, and hospital-based acute care after sepsis. Crit Care Med. 2018;46:354-60.

6. Ellerbe C, Vincent JL. Mechanisms and treatment of organ failure in sepsis. Nat Rev Nephrol. 2018;14:417-27.

7. Evans $C E$, Zhao YY. Impact of thrombosis on pulmonary endothelial injury and repair following sepsis. Am J Physiol Lung Cell Mol Physiol. 2017;312:L441-51.

8. Leligdowicz A, Richard-Greenblatt M, Wright J, Crowley VM, Kain KC. Endothelial activation: the ang/tie axis in sepsis. Front Immunol. 2018;9:838.

9. Iba T, Levy JH. Derangement of the endothelial glycocalyx in sepsis. J Thromb Haemost. 2019;17:283-94.

10. Kidney Disease: Improving Global Outcomes (KDIGO) Acute Kidney Injury Work Group. KDIGO clinical practice guideline for acute kidney injury. Kidney Int Suppl. 2012;2:1-138.

11. Peerapornratana S, Manrique-Caballero CL, Gómez H, Kellum JA. Acute kidney injury from sepsis: current concepts, epidemiology, pathophysiology, prevention and treatment. Kidney Int. 2019;96:1083-99. 
12. Peters E, Antonelli M, Wittebole $X$, Nanchal R, François B, Sakr Y, Vincent $J$, Pickkers P. A worldwide multicentre evaluation of the influence of deterioration or improvement of acute kidney injury on clinical outcome in critically ill patients with and without sepsis at ICU admission: results from The Intensive Care Over Nations audit. Crit Care. 2018;22:188.

13. Chertow GM, Burdick E, Honour M, Bonventre JV, Bates DW. Acute kidney injury, mortality, length of stay, and costs in hospitalized patients. J Am Soc Nephrol. 2005;16:3365-70.

14. Ali T, Khan I, Simpson W, Prescott G, Townend J, Smith W, Macleod A Incidence and outcomes in acute kidney injury: a comprehensive population-based study. J Am Soc Nephrol. 2007;18:1292-8.

15. Hoste EAJ, Kellum JA, Selby NM, Zarbock A, Palevsky PM, Bagshaw SM, Goldstein SL, Cerdá J, Chawla LS. Global epidemiology and outcomes of acute kidney injury. Nat Rev Nephrol. 2018;14:607-25.

16. El-Achkar TM, Dagher PC. Tubular cross talk in acute kidney injury: a story of sense and sensibility. Am J Physiol Renal Physiol. 2015;308:F1317-23.

17. Gomez H, Ince C, De Backer D, Pickkers P, Payen D, Hotchkiss J, Kellum JA. A unified theory of sepsis-induced acute kidney injury: inflammation, microcirculatory dysfunction, bioenergetics, and the tubular cell adaptation to injury. Shock. 2014;41:3-11.

18. Agrawal A, Matthay MA, Kangelaris KN, Stein J, Chu JC, Imp BM, Cortez A, Abbott J, Liu KD, Calfee CS. Plasma angiopoietin-2 predicts the onset of acute lung injury in critically ill patients. Am J Respir Crit Care Med. 2013;187:736-42.

19. Parikh SM. Targeting Tie2 and the host vascular response in sepsis. Sci Transl Med. 2016;8:335fs9.

20. Schmidt EP, Yang Y, Janssen WJ, Gandjeva A, Perez MJ, Barthel L, Zemans RL, Bowman JC, Koyanagi DE, Yunt ZX, Smith LP, Cheng SS, Overdier KH, Thompson KR, Geraci MW, Douglas IS, Pearse DB, Tuder RM. The pulmonary endothelial glycocalyx regulates neutrophil adhesion and lung injury during experimental sepsis. Nat Med. 2012;18:1217-723.

21. Yang KY, Liu KT, Chen YC, Chen CS, Lee YC, Perng RP, Feng JY. Plasma soluble vascular endothelial growth factor receptor-1 levels predict outcomes of pneumonia-related septic shock patients: a prospective observational study. Crit Care. 2011;15:R11.

22. Levy MM, Fink MP, Marshall JC, Abraham E, Angus D, Cook D, Cohen J, Opal SM, Vincent JL, Ramsay G; 2001 SCCM/ESICM/ACCP/ATS/SIS. International Sepsis Definitions Conference. Crit Care Med. 2003;31:1250-1256.

23. Wang CY, Calfee CS, Paul DW, Janz DR, May AK, Zhuo H, Bernard GR, Matthay MA, Ware LB, Kangelaris KN. One-year mortality and predictors of death among hospital survivors of acute respiratory distress syndrome. Intensive Care Med. 2014:40:388-96.

24. Chen W, Janz DR, Shaver CM, Bernard GR, Bastarache JA, Ware LB. Clinical characteristics and outcomes are similar in ARDS diagnosed by oxygen saturation/FiO2 ratio compared with $\mathrm{PaO} 2 / \mathrm{FiO} 2$ ratio. Chest. 2015;148:1477-83.

25. Knaus WA, Draper EA, Wagner DP, Zimmerman JE. APACHE II: a severity of disease classification system. Crit Care Med. 1985;13:818-29.

26. Bernard G. The Brussels score. Sepsis. 1997;1:43-4

27. Murphy LS, Wickersham N, MCNeil JB, Shaver CM, May AK, Bastarache JA, Ware LB. Endothelial glycocalyx degradation is more severe in patients with non-pulmonary sepsis compared to pulmonary sepsis and associates with risk of ARDS and other organ dysfunction. Ann Intensive Care. 2017;7:102.

28. SelfWH, Semler MW, Wanderer JP, Wang L, Byrne DW, Collins SP, Slovis CM, Lindsell CJ, Ehrenfeld JM, Siew ED, Shaw AD, Bernard GR, Rice TW, SALT-ED Investigators. Balanced crystalloids versus saline in noncritically ill adults. N Engl J Med. 2018;378:819-28.

29. Závada J, Hoste E, Cartin-Ceba R, Calzavacca P, Gajic O, Clermont G, Bellomo R, Kellum JA, AKI6 investigators. A comparison of three methods to estimate baseline creatinine for RIFLE classification. Nephrol Dial Transplant. 2010;25:3911-8.

30. ARDS Definition Task Force, Ranieri VM, Rubenfeld GD, Thompson BT, Ferguson ND, Caldwell E, Fan E, Camporota L, Slutsky AS. Acute respiratory distress syndrome: the Berlin definition. JAMA. 2012;307:2526-33.
31. Rice TW, Wheeler AP, Bernard GR, Hayden DL, Schoenfeld DA, Ware LB, National Institutes of Health, National Heart, Lung, and Blood Institute ARDS Network. Comparison of the SpO2/FiO2 ratio and the $\mathrm{PaO} 2 / \mathrm{FiO} 2$ ratio in patients with acute lung injury or ARDS. Chest. 2007;132:410-7.

32. Kümpers $P$, David S, Haubitz M, Hellpap J, Horn R, Bröcker V, Schiffer M, Haller $\mathrm{H}$, Witte T. The Tie2 receptor antagonist angiopoietin 2 facilitates vascular inflammation in systemic lupus erythematosus. Ann Rheum Dis. 2009;68:1638-43.

33. Kümpers P, Hellpap J, David S, Horn R, Leitolf H, Haller H, Haubitz M. Circulating angiopoietin-2 is a marker and potential mediator of endothelial cell detachment in ANCA-associated vasculitis with renal involvement. Nephrol Dial Transplant. 2009;24:1845-50.

34. Akwii RG, Sajib MS, Zahra FT, Mikelis CM. Role of angiopoietin-2 in vascular physiology and pathophysiology. Cells. 2019;8:471.

35. Robinson-Cohen C, Katz R, Price BL, Harju-Baker S, Mikacenic C, Himmelfarb J, Liles WC, Wurfel MM. Association of markers of endothelial dysregulation Ang1 and Ang2 with acute kidney injury in critically ill patients. Crit Care. 2016;20:207.

36. De Freitas CN, Gaudet A, Portier L, Tsicopoulos A, Mathieu D, Lassalle P. Endocan, sepsis, pneumonia, and acute respiratory distress syndrome. Crit Care. 2018;22:280

37. Ziegler T, Horstkotte J, Schwab C, Pfetsch V, Weinmann K, Dietzel S, Rohwedder I, Hinkel R, Gross L, Lee S, Hu J, Soehnlein O, Franz WM, Sperandio M, Pohl U, Thomas M, Weber C, Augustin HG, Fässler R, Deutsch $U$, Kupatt C. Angiopoietin 2 mediates microvascular and hemodynamic alterations in sepsis. J Clin Invest. 2013;123:3436-45.

38. Fisher J, Douglas JJ, Linder A, Boyd JH, Walley KR, Russell JA. Elevated plasma angiopoietin-2 levels are associated with fluid overload, organ dysfunction, and mortality in human septic shock. Crit Care Med. 2016:44:2018-27.

39. Kümpers $P$, Lukasz A, David S, Horn R, Hafer C, Faulhaber-Walter R, Fliser D, Haller $\mathrm{H}$, Kielstein JT. Excess circulating angiopoietin-2 is a strong predictor of mortality in critically ill medical patients. Crit Care. 2008;12:R147.

40. David S, Mukherjee A, Ghosh CC, Yano M, Khankin EV, Wenger JB, Karumanchi SA, Shapiro NI, Parikh SM. Angiopoietin-2 may contribute to multiple organ dysfunction and death in sepsis. Crit Care Med. 2012;40:3034-41.

41. Semler MW, Bernard GR, Aaron SD, Angus DC, Biros MH, Brower RG, Calfee CS, Colantuoni EA, Ferguson ND, Gong MN, Hopkins RO, Hough CL, Iwashyna TJ, Levy BD, Martin TR, Matthay MA, Mizgerd JP, Moss M, Needham DM, Self WH, Seymour CW, Stapleton RD, Thompson BT, Wunderink RG, Aggarwal NR, Reineck LA. Identifying clinical research priorities in adult pulmonary and critical care: NHLBI Working group report. Am J Respir Crit Care Med. 2020;202:511-23.

42. Gómez H, Kellum JA. Sepsis-induced acute kidney injury. Curr Opin Crit Care. 2016;22:546-53.

43. Husain-Syed F, Slutsky AS, Ronco C. Lung-kidney cross-talk in the critically ill patient. Am J Respir Crit Care Med. 2016;194:402-14.

44. Araújo CB, de Oliveira Neves FM, de Freitas DF, Arruda BFT, de Macêdo Filho LJM, Salles VB, Meneses GC, Martins AMC, Libório AB. Angiopoietin-2 as a predictor of acute kidney injury in critically ill patients and association with ARDS. Respirology. 2019;24:345-51.

45. Singbartl K, Bishop JV, Wen X, Murugan R, Chandra S, Filippi MD, Kellum JA. Differential effects of kidney-lung cross-talk during acute kidney injury and bacterial pneumonia. Kidney Int. 2011;80:633-44.

\section{Publisher's Note}

Springer Nature remains neutral with regard to jurisdictional claims in published maps and institutional affiliations. 